DOI 10.15593/2224-9354/2018.3.14

УДК $316.26: 331$

\title{
А.В. Праведников
}

\section{ВЛИЯНИЕ НАПРЯЖЕННОСТИ ТРУДА И ТЕМПОРАЛЬНЫХ ХАРАКТЕРИСТИК ЗАНЯТОСТИ НА БАЛАНС СЕМЬИ И РАБОТЫ}

\begin{abstract}
Изменения в структуре занятости населения, начавшиеся в период Второй мировой войны, запустили процесс массового выхода женщин на рынок оплачиваемого труда, полностью завершившийся в 1980-е годы. Модель семьи с одним работающим супругом потеряла свою популярность, место доминирующей заняла модель двухкарьерной семьи. Новая модель требовала от супругов большей гибкости, умения адаптироваться, комбинировать между собой исполнение семейных и профессиональных ролей, лучше планировать и распределять время. Описанные изменения нашли отражение в повестке социальных наук: сформировался сам термин баланса семьи и работы, стали проводиться исследования в данном направлении.

В статье представлены некоторые результаты социологического исследования восприятия баланса семьи и работы работниками промышленных предприятий, имеющих на иждивении детей, не достигших возраста 18 лет. Исследование проведено в период с июня по декабрь 2015 года на четырех промышленных предприятиях Республики Башкортостан ${ }^{1}$.

Основное внимание в статье уделено влиянию, которое оказывают на баланс семейной и профессиональной сфер жизни работников промышленных предприятий фракторы напряженности труда и темпоральных характеристик работы. На основании данных социологического исследования автором делается вывод, что основной дисбаланс в жизнь работников промышленных предприятий вносят чрезмерные запросы трудовой сферы в отношении времени индивида.

Ключевые слова: баланс семьи и работы, работники промышленных предприятий, объективные фракторы обеспечения баланса, субъективное восприятие баланса, напряженность, время.
\end{abstract}

Баланс семьи и работы - сравнительно новая исследовательская категория для социологической науки в целом и отечественной социологии в частности. Окончательное формирование термина произошло в 1980-е годы, став реакцией на выбор множества мужчин и женщин, предпочитавших работу и карьерные перспективы семье, друзьям и досуговому времяпрепровождению [1, p. 1].

Проблемная ситуация заключается в том, что часто если пара тем или иным образом смогла совместить участие обоих супругов в оплачиваемой деятельности с семейными обязанностями, ошибочно предполагается, что «баланс» достигнут. Несмотря на это, семьи и индивиды вынуждены постоянно справляться с возникающей напряженностью для того, чтобы совмещать семью и работу [2, p. 380].

\section{(C) Праведников А.В., 2018}

Праведников Александр Владимирович - аналитик отдела социально-демографических исследований здоровья населения ГБУЗ РБ «Медицинский информационно-аналитический центр», e-mail: aalexandrz@mail.ru.

1 Исследование проведено при финансовой поддержке РГНФ, грант № 15-13-02603 «Политика баланса семьи и работы на промышленных предприятиях Республики Башкортостан», руководитель проекта - д-р социол. наук Ф.Б. Бурханова). 
В качестве рабочего можно использовать определение Н. Стейбер: «Баланс семьи и работы - это довольно субъективный, перцептивный феномен. Мы можем определить его как ситуацию, возникающую в том случае, когда индивид ощущает, что его главные жизненные сферы и различные роли, которые он в них играет, совместимы друг с другом» [3, p. 470].

Говоря о социологическом анализе баланса семьи и работы, необходимо иметь в виду, что существуют различные традиции изучения ряда аспектов, из которых складывается данное явление. Избранная традиция в конечном итоге определяет весь дальнейший ход исследования, его гипотезы и интерпретацию полученных результатов.

Общепризнано, что семейные и профессиональные характеристики индивида (например, к первым относится количество детей, ко вторым - продолжительность рабочего дня и т.п.) оказывают влияние на его возможности эффективно исполнять роли в различных сферах деятельности.

Исходя из этого, видится логичным, что большой блок исследований в зарубежной социологии посвящен именно изучению влияния характеристик рабочего места на возможности гармонично сочетать исполнение семейных и трудовых обязанностей. В рамках этого направления разработаны подходы «требований и ресурсов» (job demands-resources) и «требований и контроля» (job demands-control) [4-7].

Согласно данным подходам, оплачиваемая работа требует от индивида не только выполнения определенных профессиональных функций, заставляет затрачивать время, усилия и т.д., но и предоставляет ресурсы (контроль), которыми он может оперировать для более успешного выполнения поставленных задач. Совокупность требований-ресурсов работы в значительной мере определяет возможности индивида исполнять свои семейные и профессиональные функции. С позиции сочетания семьи и работы ресурсы могут оказывать нивелирующее воздействие на дисбаланс между сферами, они также могут быть использованы в качестве одного из способов установления баланса.

Как правило, чем более сложная и квалифицированная работа, тем больше она выдвигает требований и предоставляет ресурсов, и наоборот. Однако в большинстве случаев ресурсов оказывается недостаточно, чтобы нивелировать дисбаланс. Многие исследователи сходятся во мнении, что более квалифицированная работа и руководящие посты повышают конфликтность сфер [2, 8-13].

Bсе запросы работы можно разделить на те, что требуют от индивида больших временных затрат (time-based demands), и те, что требуют значительных затрат сил, энергии, эмоций из-за напряженности выполняемой работы (strain-based demands). Работа сверхурочно, непредвиденные вызовы и пр. оказывают влияние преимущественно на временные затраты, а сложность 
работы, плохая психологическая обстановка, стресс, постоянные перегрузки на напряженность. При этом временной конфликт может порождать конфликт напряженности, и наоборот. Замечено, если один из супругов проводит на работе значительную часть времени, количество домашней работы, выпадающее на долю второго, увеличивается [3, 14].

В рамках данной статьи внимание будет сосредоточено как раз на том, какое влияние оказывает трудовая занятость на возможности работников с детьми выполнять семейные обязанности, какую роль в установлении баланса/дисбаланса вносят временные характеристики работы и ее напряженность.

Методика исследования. В ходе исследования были выборочно опрошены (метод - анкетирование) работники четырех промышленных предприятий, каждое из которых можно назвать типичным в своем секторе: это два крупных предприятия авиационной промышленности, средняя дочерняя организация крупного нефтяного предприятия и одно малое предприятие химической промышленности.

Когда речь идет о проведении эмпирического исследования, перед социологом всегда встает проблема адекватного, валидного и надежного измерения различных характеристик социальных объектов и явлений. Особенно сложным решение этой проблемы является в том случае, когда необходимо квантифицировать качественные объекты и явления, для которых не существует общепринятых шкал и измерителей. На данный момент баланс семьи и работы как раз может быть отнесен к таким объектам.

Как следует из приведенного в начале статьи определения, баланс семьи и работы определяется не только наличием объективных факторовпредпосылок для гармонизации семейной и трудовой сфер, но и субъективным восприятием индивида, т.е. тем, насколько позитивно или негативно он сам воспринимает существующий в его жизни уровень баланса между этими сферами [15, с. 52].

Композиция анкеты исследования была составлена так, чтобы максимально эффективно фиксировать как наличие в жизни индивида объективных факторов достижения баланса, так и его субъективное восприятие достигнутого баланса.

Фиксация наличия/отсутствия в жизни индивида объективных факторов производилась путем установления присутствия или отсутствия в жизни индивидов определенного перечня факторов. Основной для составления перечня объективных факторов, влияющих на достижение баланса, послужила таблица, которую О.Б. Савинская использовала в своем исследовании условий труда, повышающих возможности достижения баланса работающими женщинами с детьми [16]. В рамках интересующей темы эти вопросы в первую очередь способствовали выявлению роли темпоральных характеристик работы в установлении баланса. 
Второй исследуемый фактор - напряженность работы, также отчасти измерялся при помощи данных шкал, но существенную роль для оценки его влияния сыграли шкалы, призванные измерить субъективное восприятие (в частности, эмоциональную вовлеченность, психологический климат, напряженность труда). Для измерения баланса семьи и работы на субъективном уровне применялся как авторский инструментарий, так и набор шкал, апробированных в зарубежных исследованиях (например, шкалы К. Берли, Д. Карлсона, Бостонского центра семьи и работы) $[17,18]$.

Результаты исследования. По данным проведенного опроса, 85,8 \% женщин и 81,7 \% мужчин, работающих на промышленных предприятиях, с разной частотой оказываются перед необходимостью выбора между семьей и работой, что свидетельствует о существовании конфликта между исполнением семейных и трудовых обязанностей. Выявлено, что основным препятствием для установления баланса между исполнением семейных и трудовых обязанностей являются запросы трудовой сферы в отношении различных ресурсов индивида (время, силы, эмоции). В то же время присутствие в жизни работников промышленных предприятий серьезного конфликта обратной направленности, когда чрезмерные требования семейной сферы являются помехой для исполнения профессиональных обязанностей, зафиксировано не было.

Для выявления конкретных характеристик работы, оказывающих негативное влияние на возможности работников промышленных предприятий сочетать исполнение семейных и трудовых обязанностей, респондентам был задан вопрос «Скажите, пожалуйста, что из нижеперечисленного более всего затрудняет для Вас сочетание семьи и работы на этом предприятии?», с возможностью выбора вплоть до 5 вариантов ответа. Результаты ответов представлены в табл. 1.

Таблица 1

Факторы, негативно влияющие на сочетание семьи и работы (в \%)*

\begin{tabular}{|l|c|c|c|}
\hline \multicolumn{1}{|c|}{ Вариант ответа } & Женщины & Мужчины & Всего \\
\hline Необходимость задерживаться на работе & 48,2 & 44,2 & 45,4 \\
\hline Частые вызовы на работу по вечерам или в выходные & 7,6 & 19,8 & 14,5 \\
\hline Плохие отношения с начальством & 4,1 & 5,5 & 5,1 \\
\hline Неудобный график работы & 10,0 & 24,0 & 17,6 \\
\hline Невозможность пользоваться даже положенными льготами & 2,9 & 7,8 & 5,6 \\
\hline Неудобное время работы & 8,8 & 7,4 & 7,9 \\
\hline Невозможность планировать свой рабочий день & 8,8 & 24,0 & 18,1 \\
\hline $\begin{array}{l}\text { Отсутствие дополнительных льгот и выплат } \\
\text { для сотрудников с детьми }\end{array}$ & 16,5 & 6,5 & 11,0 \\
\hline $\begin{array}{l}\text { Негативные отношения в коллективе, стрессовая } \\
\text { обстановка }\end{array}$ & 4,1 & 6,0 & 5,4 \\
\hline Неудобное расположение работы & 21,2 & 31,8 & 27,8 \\
\hline
\end{tabular}


Окончание табл. 1

\begin{tabular}{|l|c|c|c|}
\hline \multicolumn{1}{|c|}{ Вариант ответа } & Женщины & Мужчины & Всего \\
\hline $\begin{array}{l}\text { Невозможность выполнять хотя бы часть работы } \\
\text { из дома (по телефону или через интернет) }\end{array}$ & 25,9 & 11,5 & 17,6 \\
\hline Низкая заработная плата & 22,9 & 23,5 & 23,2 \\
\hline $\begin{array}{l}\text { Невозможность отпроситься с работы } \\
\text { ни под каким предлогом }\end{array}$ & 11,2 & 19,8 & 16,8 \\
\hline Невозможность выйти на больничный & 5,9 & 2,3 & 3,8 \\
\hline Другое & 11,8 & 14,3 & 13,0 \\
\hline Всего & 210,0 & 248,4 & 232,8 \\
\hline
\end{tabular}

*Множественные ответы. Сумма ответов составляет более 100 \%.

Все представленные в перечне суждения предположительно оказывают негативное влияние на возможности достижения баланса между семьей и работой, но в рамках данной темы нас особо интересуют лишь некоторые - те, которые однозначно свидетельствует, что сочетание сфер затрудняется темпоральным фактором (например, необходимость задерживаться на работе, частые вызовы на работу по вечерам или в выходные, неудобный график работы, неудобное время работы). Другая часть суждений позволяет сделать вывод, что источником дисбаланса является фактор напряженности трудового процесса (например, плохие отношения с начальством, негативные отношения в коллективе, стрессовая обстановка, невозможность отпроситься с работы ни под каким предлогом).

При анализе полученных данных видно, что мужчины и женщины поразному воспринимают факторы, затрудняющие совмещение работы и родительства. Но представители обоих полов солидарны в одном: при работе на промышленном предприятии главным образом сочетание семьи и работы затрудняется темпоральным фактором - необходимостью задерживаться на работе (в целом по выборке - 45,4 \%). В свою очередь суждения, свидетельствующие о присутствии фактора напряженности (негативные отношения в коллективе, стрессовая обстановка, плохие отношения с начальством), выбирались респондентами довольно редко. Среди факторов напряженности работы наиболее часто выбирается «невозможность отпроситься с работы ни под каким предлогом» (в целом по выборке - 16,8 \%), но необходимо заметить, что в данном случае на частоту выбора этого варианта ответа могли оказывать влияние не только напряженность труда и отношения с руководством, но и сама специфика производственного процесса на промышленных предприятиях.

В табл. 2 представлены шесть суждений, три из которых (№ 1, 2 и 3) свидетельствуют о дисбалансе, порождаемом временным фактором, оставшиеся сигнализируют о факторе напряженности (№ 4, 5 и 6). В таблице отражена доля выборов вариантов «абсолютно верно» и «скорее верно». 
Степень согласия респондентов с некоторыми суждениями о совмещении семьи и работы (в \%)*

\begin{tabular}{|l|c|c|c|c|c|c|}
\hline \multicolumn{1}{|c|}{ Формулировка суждения } & \multicolumn{2}{|c|}{ Женщины } & \multicolumn{2}{|c|}{ Мужчины } & \multicolumn{2}{|c|}{$\begin{array}{c}\text { В целом } \\
\text { по выборке }\end{array}$} \\
\cline { 2 - 8 } & $\begin{array}{c}\text { Абс. } \\
\text { верно }\end{array}$ & $\begin{array}{c}\text { Скорее } \\
\text { верно }\end{array}$ & $\begin{array}{c}\text { Абс. } \\
\text { верно }\end{array}$ & $\begin{array}{c}\text { Скорее } \\
\text { верно }\end{array}$ & $\begin{array}{c}\text { Абс. } \\
\text { верно }\end{array}$ & $\begin{array}{c}\text { Скорее } \\
\text { верно }\end{array}$ \\
\hline $\begin{array}{l}\text { 1. Для руководства важнее всего, } \\
\text { чтобы работа была сделана, неважно - } \\
\text { в рабочее время или сверхурочно }\end{array}$ & 49,7 & 29,5 & 62,3 & 23,4 & 57,4 & 25,7 \\
\hline $\begin{array}{l}\text { 2. На нашем предприятии принято } \\
\text { работать сверхурочно }\end{array}$ & 24,9 & 20,4 & 15,8 & 14,6 & 19,7 & 16,9 \\
\hline $\begin{array}{l}\text { 3. Если хочешь получить продвижение } \\
\text { по службе, тебе нужно много работать, } \\
\text { в том числе сверхурочно }\end{array}$ & 21,3 & 32,2 & 20,7 & 37,2 & 20,9 & 35,6 \\
\hline $\begin{array}{l}\text { 4. Сотрудники чувствуют недовольст- } \\
\text { во, если кто-то из коллег пользуется } \\
\text { возможностями по совмещению семьи } \\
\text { и работы, ведь в этом случае им при- } \\
\text { ходится больше работать }\end{array}$ & 6,1 & 16,0 & 4,6 & 16,0 & 5,2 & 15,8 \\
\hline $\begin{array}{l}\text { 5. Начальство холодно относится } \\
\text { к сотрудникам, пользующимся мерами } \\
\text { по совмещению семьи и работы }\end{array}$ & 2,2 & 13,0 & 0,8 & 5,4 & 1,4 & 8,6 \\
\hline $\begin{array}{l}\text { 6. Приоритет семьи над работой в } \\
\text { жизни сотрудника негативно скажется } \\
\text { на его карьере на нашем предприятии }\end{array}$ & 9,4 & 27,6 & 5,5 & 34,9 & 7,1 & 31,4 \\
\hline
\end{tabular}

* Вместе с ответами «И верно и не верно», «Скорее не верно» и «Абсолютно не верно» сумма ответов по строке отдельно для женщин и мужчин составляет $100 \%$.

При ответе на данный вопрос респондентам предлагалось выразить степень своего согласия с представленными суждениями. Полученные результаты подтверждают, что темпоральные характеристики работы оказывают большее влияние на возникновение дисбаланса в жизни работников. В целом по выборке суммарное согласие с первым суждением выразили 79,2 \% респондентов, со вторым - 45,3 \%, с третьим - 53,5 \%. В случае с суждениями, свидетельствующими о факторе напряженности, максимальная доля согласных составила $37 \%$.

Bblводb. Для большинства опрошенных причиной конфликта между семьей и работой являются чрезмерные требования профессиональной сферы. При этом необходимо конкретизировать, что основным препятствием для достижения баланса выступают запросы трудовой сферы в отношении времени индивида. Фактор напряженности также присутствует, но оказывает значительно меньшее влияние на возможности работников промышленных предприятий гармонизировать сочетание семейных и трудовых обязанностей. 
Именно временной ресурс является ключевым в вопросах совмещения ввиду его полной невосполнимости, следовательно, если какая-либо из сфер предъявляет завышенные требования в отношении времени индивида, это всегда в значительной мере дисгармонизирует его жизнь.

Можно утверждать, что результаты исследования согласуются с выводами других исследователей, большинство из которых, не умаляя влияния вневременных характеристик, все же сходятся во мнении, что именно темпоральные характеристики работы оказывают наибольшее влияние на установление баланса между сферами $[11,12,19,20]$.

\section{Список литературы}

1. Abhilasha Joshi Sharma. Balance between Work and Life: Challenges and Solutions // International Journal of Applied Research and Studies (iJARS). 2013. - Vol. 2, iss. 11. - P. 1.

2. Crompton R., Lyonette C. Work-Life «Balance» in Europe // Acta Sociologica. - 2006. - № 49. - P. 379-393.

3. Steiber N. Reported Levels of Time-based and Strain-based Conflict Between Work and Family Roles in Europe: A Multilevel Approach // Social Indicators Research. - 2009. - Vol. 93, iss. 3. - P. 469-488.

4. Bakker A.B., Demerouti E. The Job Demands-Resources model: State of the art // Journal of Managerial Psychology. - 2007. - Vol. 22. - P. 309-328.

5. The job demands-resources model of burnout / E. Demerouti, A.B. Bakker, F. Nachreiner, W.B. Schaufeli // Journal of Applied Psychology. - 2001. - Vol. 86, iss. 3. - P. 499-512.

6. Karasek R. A Job demands, job decision latitude, and mental strain: Implications for job redesign // Administrative Science Quarterly. - 1979. - Vol. 24. P. 285-308.

7. Voydanoff $P$. Toward a conceptualization of perceived work-family fit and balance: A demands and resources approach // Journal of Marriage and Family. 2005. - Vol. 67, iss. 4. - P. 822-846.

8. Gallie D., Russell H. Work-Family Conflict and Working Conditions in Western Europe // Social Indicators Research. - 2009. - Vol. 93, iss. 3. - P. 445-467.

9. Glavin P., Schieman S. Work-Family Role Blurring and Work-Family Conflict: The Moderating Influence of Job Resources and Job Demands // Work and Occupations. - 2012. - Vol. 31, iss. 1. - P. 71-98.

10. Jacobs J.A, Gerson K. Overworked Individuals or Overworked Families? Explaining Trends in Work, Leisure, and Family Time // Work and Occupations. 2001. - Vol. 28, iss. 1. - P. 40-63.

11. Laurijssen I., Glorieux I. Balancing Work and Family: A Panel Analysis of the Impact of Part-Time Work on the Experience of Time Pressure // Social Indicators Research. - 2013. - Vol. 112, iss. 1. - P. 1-17. 
12. Ruppanner L. Conflict Between Work and Family: An Investigation of Four Policy Measures // Social Indicators Research. - 2013. - Vol. 110. - P. 327-347.

13. Voydanoff P. Work, family, and community: Exploring interconnections. Mahwah. - N.Y.: Routledge, 2007.

14. Greenhaus J.H., Beutell N.J. Sources of conflict between work and family roles // Academy of Management Review. - 1985. - Vol. 10, iss. 1. - P. 76-88.

15. Бурханова Ф.Б., Праведников А.В. Влияние темпоральных характеристик трудовой занятости на баланс семьи и работы // Вестник ВЭГУ. Научный журнал по социально-экономическим и гуманитарным наукам. - 2016. № 2(82). - С. 51-60.

16. Савинская О.Б. Баланс работы и семьи: стратегии совмещения профессиональных и семейных обязанностей работающими матерями в Москве // Журнал социологии и социальной антропологии. - 2013. - T. XVI, № 2 (67). С. 142-167.

17. Burley K. Work-family conflict and marital adjustment in dual career couples: A comparison of three time models: Unpublished doctoral dissertation. Claremont Graduate school, Claremont, CA, 1989.

18. Carlson D.S., Kacmar K.M., Williams L.J. Construction and initial validation of a multi-dimensional measure of work-family conflict // Journal of Vocational Behavior. - 2000. - Vol. 56, iss. 2. - P. 249-276.

19. Berger E.M. Maternal employment and happiness: The effect of nonparticipation and part-time employment on mothers' life satisfaction. SOEP paper № 178 (on Multidisciplinary Panel Data Research). - Berlin: Deutsches Institut fur Wirtschaftsforschung, 2009. - 28 p.

20. Lippe T., Jager A., Kops Y. Combination Pressure. The Paid WorkFamily Balance of Men and Women in European Countries // Acta Sociologica. 2006. - Vol. 49, iss. 3. - P. 303-319.

\section{References}

1. Abhilasha J.S. Balance between work and life: challenges and solutions. International Journal of Applied Research and Studies, Nov. 2013, vol. 2, iss. 11, p. 1.

2. Crompton R., Lyonette C. Work-life "balance" in Europe. Acta Sociologica, 2006, no. 49, pp. 379-393.

3. Steiber N. Reported levels of time-based and strain-based conflict between work and family roles in Europe: a multilevel approach. Social Indicators Research, 2009, vol. 93, iss. 3, pp. 469-488.

4. Bakker A.B, Demerouti E. The job demands-resources model: state of the art. Journal of Managerial Psychology, 2007, vol. 22, pp. 309-328.

5. Demerouti E., Bakker A.B., Nachreiner F., Schaufeli W.B. The job demands-resources model of burnout. Journal of Applied Psychology, 2001, vol. 86, iss. 3, pp. 499-512. 
6. Karasek R. A job demands, job decision latitude, and mental strain: implications for job redesign. Administrative Science Quarterly, 1979, vol. 24, pp. 285-308.

7. Voydanoff P. Toward a conceptualization of perceived work-family fit and balance: a demands and resources approach. Journal of Marriage and Family, 2005, vol. 67, iss. 4, pp. 822-846.

8. Gallie D., Russell H. Work-family conflict and working conditions in western Europe. Social Indicators Research, 2009, vol. 93, iss. 3, pp. 445-467.

9. Glavin P., Schieman S. Work-family role blurring and work-family conflict: the moderating influence of job resources and job demands. Work and Occupations, 2012, vol. 31, iss. 1, pp. 71-98.

10. Jacobs J.A., Gerson K. Overworked individuals or overworked families? Explaining trends in work, leisure, and family time. Work and Occupations, 2001, vol. 28 , iss. 1, pp. 40-63.

11. Laurijssen I., Glorieux I. Balancing work and family: a panel analysis of the impact of part-time work on the experience of time pressure. Social Indicators Research, 2013, vol. 112, iss. 1, pp. 1-17.

12. Ruppanner L. Conflict between work and family: an investigation of four policy measures. Social Indicators Research, 2013, vol. 110, pp. 327-347.

13. Voydanoff P. Work, family, and community: exploring interconnections. Mahwah, NJ, Routledge, 2007.

14. Greenhaus J.H., Beutell N.J. Sources of conflict between work and family roles. Academy of Management Review, 1985, vol. 10, iss. 1, pp. 76-88.

15. Burkhanova F.B., Pravednikov A.V. Vliianie temporal'nykh kharakteristik trudovoi zaniatosti na balans sem'i i raboty [Influence of temporal characteristics of employment on family and work balance]. Vestnik VEGU, 2016, no. 2(82), pp. 51-60.

16. Savinskaia O.B. Balans raboty i sem'i: strategii sovmeshcheniia professional'nykh i semeinykh obiazannostei rabotaiushchimi materiami v Moskve [Work-family balance: how working mothers in Moscow reconcile their work and maternity]. The Journal of Sociology and Social Anthropology, 2013, vol. 16, no. 2 (67), pp. 142-167.

17. Burley K. Work-family conflict and marital adjustment in dual career couples: a comparison of three time models: unpublished doctoral dissertation. Claremont, CA, Claremont Graduate school, 1989.

18. Carlson D.S., Kacmar K.M., Williams L.J. Construction and initial validation of a multi-dimensional measure of work-family conflict. Journal of Vocational Behavior, 2000, vol. 56, iss. 2, pp. 249-276.

19. Berger E.M. Maternal employment and happiness: the effect of nonparticipation and part-time employment on mothers' life satisfaction. SOEP paper no. 178 (on Multidisciplinary Panel Data Research). Berlin, Deutsches Institut fur Wirtschaftsforschung, 2009, 28 p. 
20. Lippe T., Jager A., Kops Y. Combination pressure. The paid work-family balance of men and women in European countries. Acta Sociologica, 2006, vol. 49, iss. 3, pp. 303-319.

Оригинальность $79 \%$

Получено 20.01.2018 Принято 20.02.2018 Опубликовано 28.09.2018

\title{
A.V. Pravednikov \\ INFLUENCE OF LABOUR INTENSITY AND TEMPORAL PARAMETERS OF EMPLOYMENT ON WORK-FAMILY BALANCE
}

\begin{abstract}
Changes in the structure of employment, which began during the Second World War, launched the mass process of women's joining the market of paid labor, which was complete in the 1980ies. The model of a family with only one working spouse lost its popularity, the dominant position now taken by dual-wage earner family model. The new model demanded from spouses more flexibility, an ability to adapt, to combine the performance of family and occupational roles, to better plan and allocate time. The described changes were reflected in social sciences agenda: the term of work-family balance was coined, and studies in this avenue started.

The article presents some results of a sociological study on perception of work-family balance of workers in industrial enterprises with dependent children under the age of 18. The research was conducted from June to December 2015 at four industrial enterprises of the Republic of Bashkortostan.

The main attention in the article is given to the influence of labor intensity factors and working temporal characteristics on the balance between family and professional spheres of the lives of industrial enterprises' workers. At the end of the article, the author concludes that the main imbalance in industrial workers' life is made by excessive demands of the labor sphere regarding personal time.

Keywords: work-family balance, workers of industrial enterprises, objective factors of ensuring the balance, subjective balance perception, intensity, time.
\end{abstract}

Aleksandr V. Pravednikov - Analyst, Department of Socio-Demographic Research of Public Health, Medical Information and Analytical Center, e-mail: aalexandrz@mail.ru.

Received 20.01.2018 Accepted 20.02.2018 Published 28.09.2018 